\title{
ATM Proficient Tumor
}

National Cancer Institute

\section{Source}

National Cancer Institute. ATM Proficient Tumor. NCI Thesaurus. Code C163009.

A finding indicating that the expression and activity of AT M in a tumor sample is detected at or near normal levels. 